\title{
Treatment for mild cognitive impairment: systematic review
}

Claudia Cooper, Ryan Li, Constantine Lyketsos and Gill Livingston

\section{Background}

More people are presenting with mild cognitive impairment $(\mathrm{MCl})$, frequently a precursor to dementia, but we do not know how to reduce deterioration.

\begin{abstract}
Aims
To systematically review randomised controlled trials (RCTs) evaluating the effects of any intervention for $\mathrm{MCl}$ on cognitive, neuropsychiatric, functional, global outcomes, life quality or incident dementia.
\end{abstract}

\section{Method}

We reviewed 41 studies fitting predetermined criteria, assessed validity using a checklist, calculated standardised outcomes and prioritised primary outcome findings in placebo-controlled studies.

\section{Results}

The strongest evidence was that cholinesterase inhibitors did not reduce incident dementia. Cognition improved in single trials of: a heterogeneous psychological group intervention over 6 months; piribedil, a dopamine agonist over 3 months; and donepezil over 48 weeks. Nicotine improved attention over 6 months. There was equivocal evidence that Huannao Yicong improved cognition and social functioning

\section{Conclusions}

There was no replicated evidence that any intervention was effective. Cholinesterase inhibitors and rofecoxib are ineffective in preventing dementia. Further good-quality RCTs are needed and preliminary evidence suggests these should include trials of psychological group interventions and piribedil.

\section{Declarations of interest}

C.L. has received grant support (research or continuing medical education) from NIMH, NIA, Associated Jewish Federation of Baltimore, Weinberg Foundation, Forest, GlaxoSmithKline, Eisai, Pfizer, Astrazeneca, Lilly, OrthoMcNeil, Bristol-Myers Squibb, Novartis, National Football League (NFL), Elan, Functional Neuromodulation; and has been a consultant/advisor to AstraZeneca, GlaxoSmithKline, Eisai, Novartis, Forest, Supernus, Adlyfe, Takeda, Wyeth, Lundbeck, Merz, Lilly, Pfizer, Genentech, Elan, NFL Players Association, NFL Benefits Office, Avanir, Zinfandel, BristolMyers Squibb; and received honorarium or travel support from Pfizer, Forest, GlaxoSmithKline, Health Monitor.
Mild cognitive impairment (MCI) is a heterogeneous state between normal ageing and early dementia. It has been referred to as an objective cognitive complaint for age, in a person with essentially normal functional activities, who does not have dementia. ${ }^{1}$ It affects $19 \%$ of people aged 65 and over. ${ }^{2}$ Around $46 \%$ of people with MCI develop dementia within 3 years compared with $3 \%$ of the population of the same age. ${ }^{3}$ Petersen distinguishes subtypes, depending on whether single or multiple cognitive domains are affected, and whether there is a predominant memory complaint. Amnestic MCI, in which memory is affected, more often progresses to Alzheimer's disease, whereas MCI affecting a single non-memory domain may herald frontotemporal or Lewy body dementia. A vascular aetiology is more likely in multiple domain $\mathrm{MCI} .{ }^{1}$ Thus, a group of people with MCI may differ from each other, clinically and neuropathologically.

The number of individuals diagnosed with $\mathrm{MCI}$ is growing in Western countries as people are encouraged to present early with memory problems to avoid crisis, but we know little about how to treat it. The UK National Institute for Health and Care Excellence (NICE) recommends follow-up to ensure dementia is diagnosed and care planned at an early stage, but does not mention specific treatments. ${ }^{4}$ Jorm et al ${ }^{5}$ calculated that dementia prevalence would be halved if its onset were delayed for 5 years. Neuroprotection, treating vascular risk factors or increasing cognitive reserves could theoretically delay dementia, and could be targeted at people with MCI who are at a particularly high risk of developing it. Previous reviews have focused on specific treatments for MCI. Systematic reviews of randomised controlled trials (RCTs) of all cholinesterase inhibitors, ${ }^{6}$ donepezil ${ }^{7}$ and galantamine ${ }^{8}$ concluded that there are marginal beneficial effects that are outweighed by the risks of adverse events, including an unexplained increased mortality rate with galantamine. A 2009 Cochrane review found that memory training (specific neuropsychological exercises to improve memory) improved immediate and delayed verbal recall in people with MCI compared with no treatment, but there were no effects when compared with an active control group. ${ }^{9}$ More recent reviews have included RCT and non-RCT studies and suggested that cognitive interventions may improve memory for specific information, with less evidence that they improve overall cognition. ${ }^{10-13}$ We aimed to carry out the first systematic review of all types of intervention for MCI, to identify the best current treatment evidence.

\section{Method}

\section{Search strategy and selection criteria}

We searched PubMed (1946-), Web of Science (1900-), Cochrane Systematic Reviews Database (c.1993-), PsycINFO (1880-), CINAHL (1937-) and AMED (1985-) through to 10 July 2012 (updated 27 January 2013), using the words: "mild cognitive", "cognitive impairment", "benign senescent forgetfulness" OR "age associated cognitive decline" AND treatment AND (controlled trial OR RCT). No limits were applied for language or time published. We searched references of included papers and systematic reviews identified in the search and contacted experts. We included RCTs evaluating any treatment for MCI that reported as an outcome: cognition (specific domains or global), conversion to dementia; functional, behavioural, quality of life or global measures. We included studies where all participants or a separately analysed subgroup had MCI. 


\section{Data extraction}

One author (C.C.) extracted study characteristics (online Tables DS1 and DS2). We contacted two authors to request unreported data; and obtained this for one ${ }^{14}$ but not the second study. ${ }^{15}$

To assess risk of bias, two authors (C.C. and R.L.) independently evaluated study validity using questions adapted from the Critical Appraisal Skills Programme (CASP; www.hello.nhs.uk/documents/ CAT6-Randomised_Controlled_Trials.pdf).

(a) Were participants appropriately allocated to intervention and control groups? (Was randomisation independent?)

(b) Were patients and clinicians, as far as possible, 'masked' to treatment allocation?

(c) Were all patients who entered the trial accounted for and an intention-to-treat analysis used?

(d) Were all participants followed up and data collected in the same way?

(e) Was a power calculation carried out, based on one of our outcomes of interest?

Disagreements were resolved by consensus between authors.

\section{Analysis}

We compared control and intervention groups post-intervention. We prioritised results from placebo-controlled studies that identified one or two primary outcomes, as these were less likely to have reported significant chance findings. For primary outcome results we displayed standardised outcomes in Forest plots (standardised mean difference (SMD), standardised mean change (SMC), hazard ratios (HR) or odds ratio (OR)) for primary outcomes using statsdirect statistical software version 2.7.9 on Windows; ${ }^{16}$ for some studies where these results were unavailable we calculated SMD or SMC from mean (or mean change), appropriate standard deviations and $n$ for intervention and control groups postintervention. Our calculations sometimes indicated a significant between-group difference where the authors' multivariate calculations did not, or vice versa, and we have indicated in the text where this occurred. For all other results we tabulated statistical comparisons between groups, and for the few studies where groups were not directly compared calculated SMD as above. We planned to meta-analyse results where three or more studies with comparable interventions reported comparable outcomes.

\section{Results}

Figure 1 shows a PRISMA diagram describing the results of our search strategy. We included 41 unique studies and listed excluded studies in online supplement DS1. Five Chinese studies were translated by R.L.; the remainder were published in English.

\section{Validity}

Table DS1 describes the 20 (49\%) studies that identified one or two primary outcomes: 16 were placebo-controlled and 13 used intention-to-treat analyses. Other studies are displayed in Table DS2. We rated the overall validity of studies (see methods). In total, 11 of 20 studies that identified primary outcomes and 5 of 23 studies that did not had validity scores of 4 or 5 , the highest levels of evidence.

\section{Description of studies}

Included studies recruited people with MCI via clinics or clinician referrals, ${ }^{17-28}$ advertisements, ${ }^{24,29-34}$ screening older

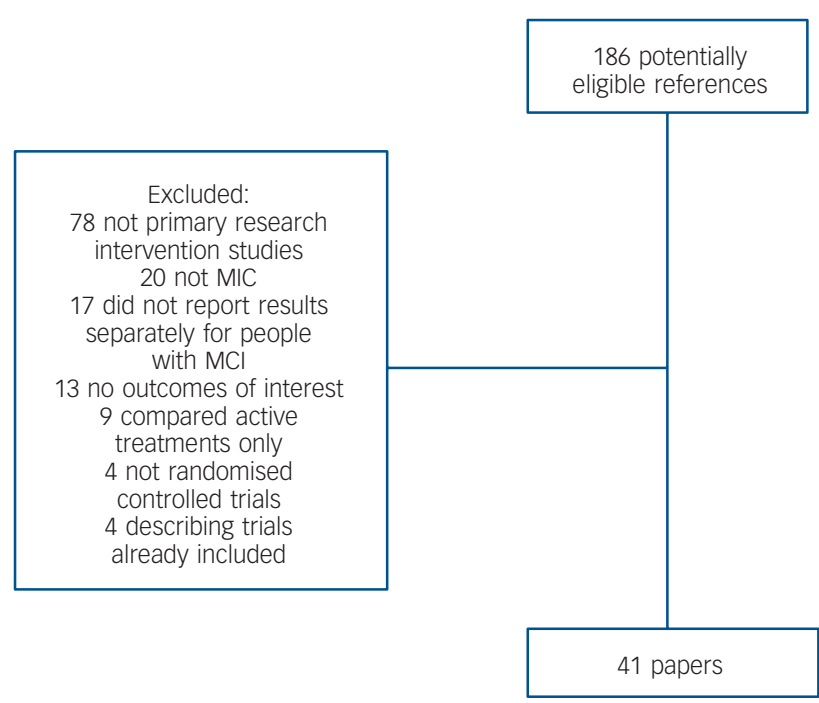

Fig. 1 Details of search strategy

$\mathrm{MCl}$, mild cognitive impairment.

populations, ${ }^{21,35-38}$ care homes, ${ }^{23,39-41}$ the local Alzheimer's society, ${ }^{34}$ pre-existing research registers, ${ }^{31,42}$ a rehabilitation centre $^{43}$ or a welfare institution. ${ }^{25}$ Several did not report the source of participants ${ }^{11,15,44-52}$ Tables DS1 and DS2 describe funding sources, inclusion criteria, sample sizes, comparators and the duration of studies. Figures 2-5 report results on all primary outcomes for which standardised outcomes could be calculated; this was not possible for four studies ${ }^{14,21,51,53}$ because data were not available or did not approximate the normal distribution, so these results are described in the text. In Table DS2, we report statistically significant between-group differences from studies without primary outcomes. Non-significant findings for all studies are in Tables DS3 and DS4. The only intervention evaluated in more than two studies was donepezil; three donepezil trials included the Alzheimer's Disease Assessment Scale-cognitive subscale (ADAS-Cog) at 6 months, but as data were not available from one, we could not meta-analyse these findings.

\section{Findings on primary outcomes in placebo-controlled studies}

Cognition improved in small studies of group memory training, cognitive stimulation and reminiscence over 6 months; and piribedil, a dopamine receptor agonist over 3 months. ${ }^{23}$ In a large, adequately powered study, donepezil improved cognition compared with placebo with 48 weeks' treatment. ${ }^{15}$ Nicotine patches improved attention in a small study of non-smokers over 6 months which was adequately powered to detect a difference on this outcome. ${ }^{21}$ Huannao Yicong, a Chinese herbal preparation containing ginseng, demonstrated efficacy on a measure of cognition and social functioning over 8 weeks in a small per protocol, responder analysis, but not when mean scores were compared between groups. ${ }^{28}$

The only finding replicated on primary outcome measures involved galantamine ${ }^{51}$ (in two studies) and other cholinesterase inhibitors (in two studies ${ }^{31,45}$ ) that did not prevent conversion to dementia. In adequately powered trials, conversion to dementia was also not reduced by vitamin $\mathrm{E}^{45}$ or Gingko biloba. ${ }^{35}$ Rofecoxib, in an adequately powered study increased dementia 
Mixed-group intervention (ADAS-Cog) ${ }^{19}$

Mixed-group intervention (MMSE) ${ }^{19}$

Fish oils (ADAS-Cog) $)^{29}$

Fish oils $(\mathrm{CIBIC})^{29}$

Donepezil (ADAS-Cog) $)^{15}$

Donepezil (CDR-SB) $)^{15}$

Triflusal (ADAS-Cog) $)^{44}$

Walking group, men (AVLT) ${ }^{38,54}$

Walking group, women (AVLT) 38,54

$B$ vitamins, men (AVLT) 38,54

$B$ vitamins, women (AVLT) $)^{38,54}$

Family intervention (prospective memory, $\mathrm{PI})^{20}$

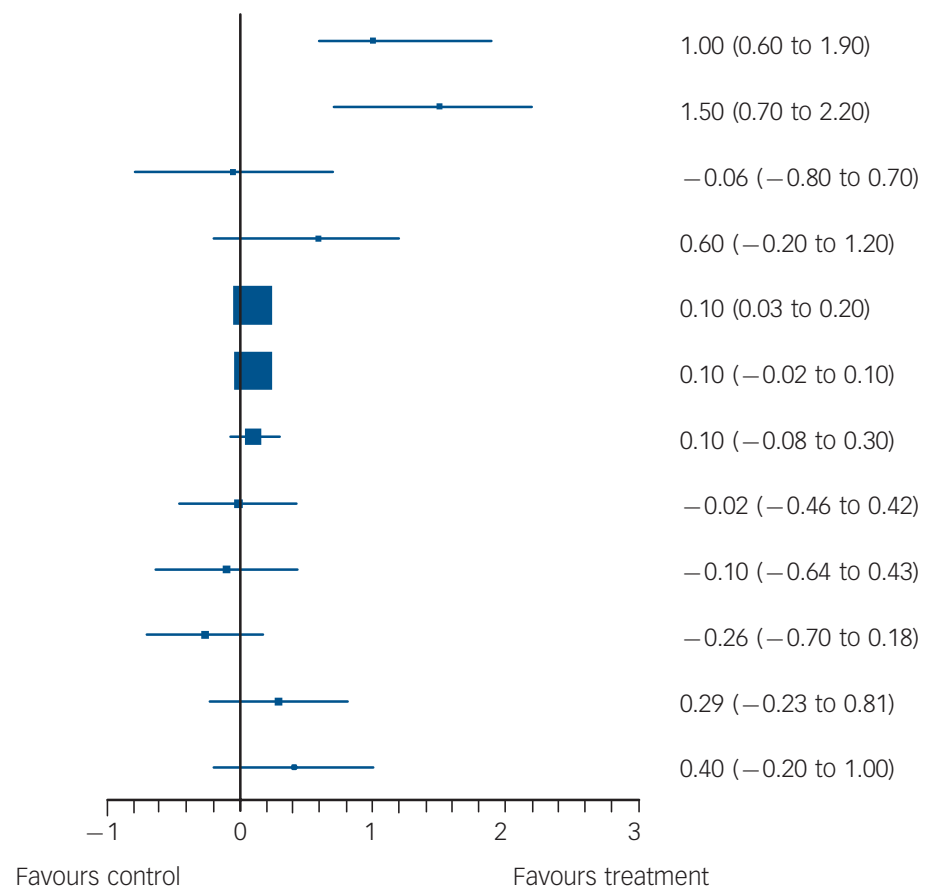

Fig. 2 Forest plots showing between-group comparisons for studies with outcomes expressed as standardised mean difference (with $95 \%$ confidence intervals).

ADAS-Cog, Alzheimer's Disease Assessment Scale-cognitive subscale; MMSE, Mini-Mental State Examination; CIBIC, Clinician's Interview-Based Impression of Change; CDR-SB , Clinical Dementia Rating scale - Sum of Boxes; AVLT, Rey Auditory Verbal Learning Test; PI, post-intervention.

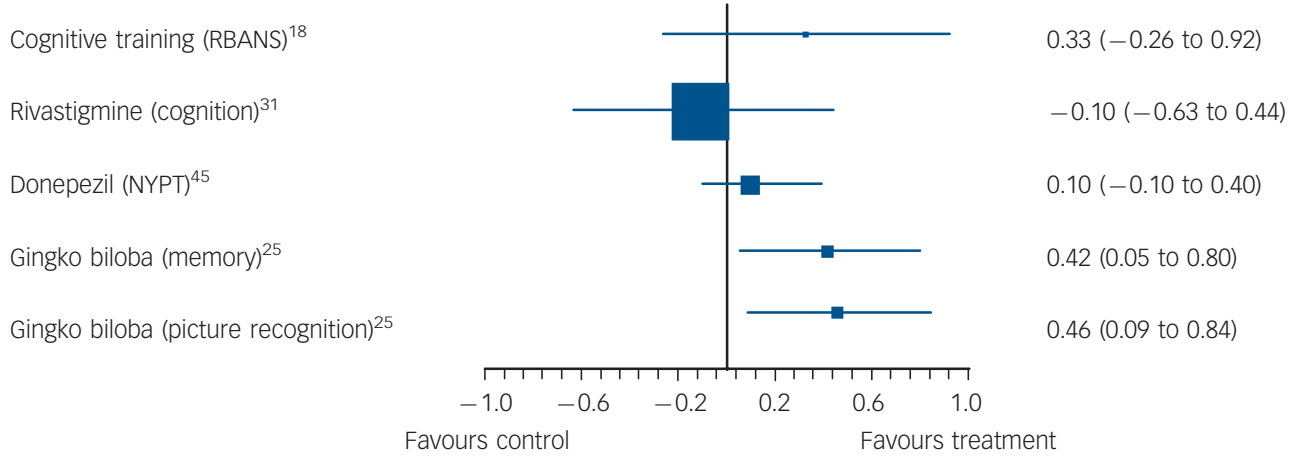

Fig. 3 Forest plots showing between-group comparisons for studies with outcomes expressed as standardised mean change from baseline (with $95 \%$ confidence intervals).

RBANS, Repeatable Battery for Assessment of Cognitive Status; NYPT, New York Paragraph Test.

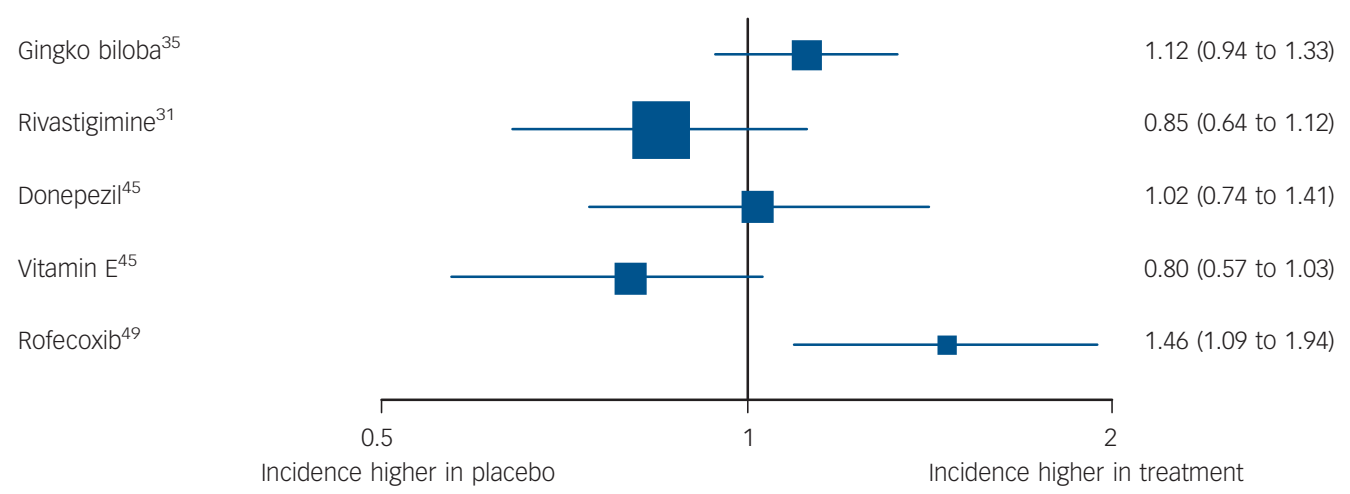

Fig. 4 Forest plots showing between-group comparisons for studies reporting hazard ratios $(95 \% \mathrm{Cl})$ for incident dementia or Alzheimer's disease (log scale). 


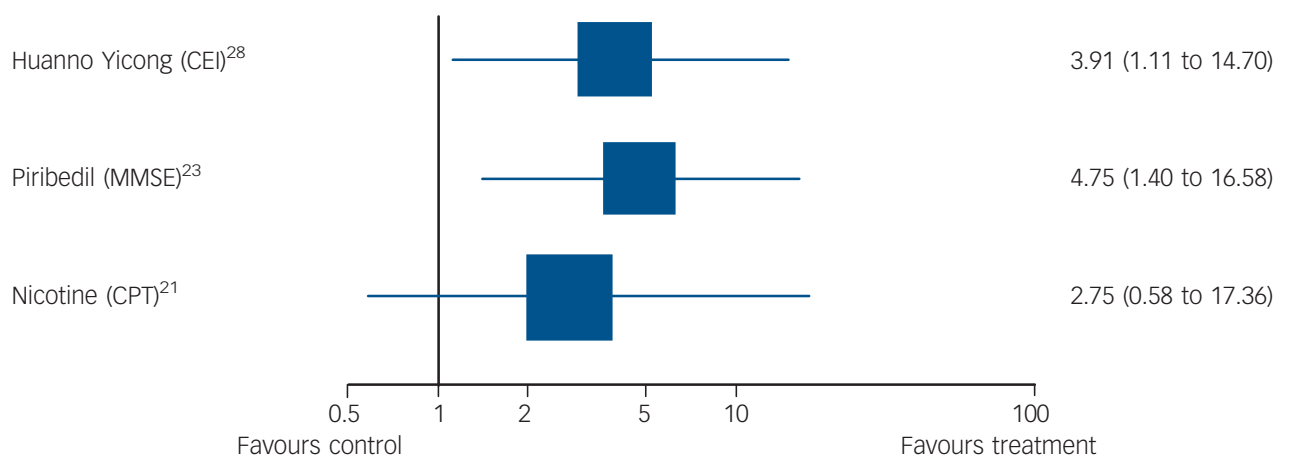

Fig. 5 Forest plots showing between-group comparisons for studies for which outcomes expressed as odds ratio for response (95\% confidence intervals) (log scale).

CEI, Cognitive Effect Index; MMSE, Mini-Mental State Examination; CPT, Connors Cognitive Performance Test.

incidence. ${ }^{49}$ Cognitive score was not improved by: 2 years of rivastigmine or 13 months of the non-steroidal anti-inflammatory drug (NSAID) triflusal ${ }^{44}$ or, in underpowered studies, by 6 weeks of computerised cognitive training ${ }^{18}$ or docosahexaenoic acid and eicosapentaenoic acid taken for 6 months; ${ }^{29}$ or a moderateintensity walking programme compared with low-intensity relaxation, balance and flexibility exercises. ${ }^{54}$

\section{Non-pharmacological interventions}

\section{Computer-assisted cognitive training (three studies)}

All three studies were probably underpowered so although results were not promising there was insufficient evidence to draw conclusions about its efficacy. Only Barnes et al ${ }^{18}$ specified primary outcomes (validity score: 4). They evaluated a programme that involved distinguishing between similar sounding words and matching sentences with pictures, for $100 \mathrm{~min}$ daily, 5 days a week. The control group listened to audio books, read an online newspaper and played a computer game. There was no significant difference between groups on the primary outcome, the Repeatable Battery for Assessment of Cognitive Status (RBANS) after 6 weeks (Fig. 3). The only significant difference, favouring the intervention, was on the delayed memory RBANS subscale (SMD $=0.53,95 \%$ CI $0.05-1.10$ ).

Two studies tested computer-assisted training, but did not specify primary outcomes. Finn \& McDonald ${ }^{55}$ evaluated a 2-month programme in a higher-quality study (validity score: 4), and Rozzini et $a l^{47}$ a 9-month programme (validity score: 3). In both studies, most results were not significant; only results for the visual attention CANTAB (Cambridge Automated Neuropsychiatric Test Assessment Battery) subscale ${ }^{55}$ and short story recall ${ }^{47}$ favoured the interventions (Table DS2).

Summary. Global cognition did not improve with cognitive training in two trials, ${ }^{18,47}$ in one of which it was a primary outcome, and there were no consistently significant findings on other secondary outcomes. Studies were all underpowered.

\section{Longer-term group psychological interventions (two studies)}

Two lower-quality studies tested 6-month group interventions. Results were conflicting. Buschert et al ${ }^{19}$ (validity score: 3 and with primary outcomes specified) tested a manualised memory training and cognitive stimulation programme, consisting of 20 $2 \mathrm{~h}$ weekly group sessions comparing 10 people in the intervention group with 12 in the control group. The memory training used mnemonics, calendars, notes and prompts; face-name association and errorless learning. In errorless learning, information was repeated frequently to avoid recall mistakes, with repetitions becoming further apart with successful recalls (spaced retrieval). The programme also included reminiscence, psychomotor and recreational tasks (for example playing with balloons), multisensory stimulation and social interaction. Participants did homework, which carers were encouraged to support. The control group met monthly and did paper-and-pencil exercises. Only participants who attended at least half of sessions were included in analyses. Global cognition, the primary outcome, improved in the intervention group in our univariate (Table DS1) and the authors' analyses that controlled for baseline score, age and educational status on both primary outcome measures. Montgomery-Åsberg Depression Rating Scale scores were also lower in the intervention compared with the control group adjusting for these factors (mean 0.7 (s.d. =1.3) for treatment group and 3.8 (s.d. $=6.1$ ) for controls, $F(1,18)=8.8, P<0.01$ ).

Troyer $e t a l^{24}$ in a small study (validity score: 1 ), evaluated 10 $2 \mathrm{~h}$ sessions, including psychoeducation, recreation, memory training and strategies, relaxation and directing to community resources. The authors found no significant differences between groups post-intervention on several measures of recall (Table DS4).

\section{Summary.}

(a) Twenty sessions of memory training, reminiscence, cognitive stimulation, psychomotor recreation and social interaction improved global cognition on a primary outcome in a single, very small, 6-month placebo-controlled trial that did not carry out an intention-to-treat analysis. ${ }^{19}$

(b) Ten sessions of memory training, psychoeducation and relaxation did not improve recall on secondary outcomes in one small 6-month trial. ${ }^{24}$

\section{Short-term (6-week) psychological group interventions (two studies)}

Both studies were lower quality (validity score: 2) and were underpowered so although neither improved memory there was insufficient evidence to reject this intervention type. Unverzagt et $a l^{14}$ specified primary outcomes and compared three interventions, all of ten sessions teaching specific cognitive strategies, with a no-treatment control. The interventions were: memory training strategies; reasoning training; and processing speed 
training. Booster training was provided to $60 \%$ of participants, approximately 11 months later. Memory training participants did not improve post-intervention, or 1 or 2 years later on the composite memory measure $v$. those receiving no treatment $(\mathrm{SMD}=-0.01,95 \% \mathrm{CI}-0.18$ to $-0.10, P>0.05)$. Participants receiving the processing speed intervention improved on processing speed $(\mathrm{SMD}=-1.4,95 \% \mathrm{CI}-1.1$ to $-0.8, P<0.001)$ and the reasoning group participants improved on reasoning post-intervention $(\mathrm{SMD}=0.57, P<0.001)$ and 2 years $(\mathrm{SMD}=0.28$, $P<0.05)$, but not 1 year later $(\mathrm{SMD}=021, P>0.05)$, compared with those receiving no treatment.

Rapp et al $^{46}$ did not specify primary outcomes. They evaluated six weekly $2 \mathrm{~h}$ sessions, of psychoeducation, relaxation, memory strategies (cueing, categorisation, chunking, method of loci) and homework. Participants received a manual, which was also sent to the control group who otherwise had no treatment. There were no significant differences between groups on several memory measures, post-intervention or 6 months later.

\section{Summary.}

(a) Memory did not improve over 6 weeks in two short-term, underpowered group intervention trials teaching memory strategies, which were not placebo-controlled. ${ }^{14,46}$

(b) Specific interventions to improve reasoning and processing speed respectively significantly improved these primary outcomes, in an underpowered single, non-placebo-controlled trial. $^{14}$

\section{Family psychological interventions (one study)}

This lower-quality study indicated that a family psychological intervention might improve prospective memory. Kinsella et al ${ }^{20}$ (validity score: 2 ) compared a course of five weekly $1.5 \mathrm{~h}$ family intervention sessions to a waiting list control. Sessions involved problem-solving everyday memory difficulties and practising possible strategies. Written session material was provided. Results on the primary outcome, an unvalidated (to our knowledge) prospective memory test favoured the intervention 2 weeks and 4 months post-intervention, controlling for baseline scores and age; the SMD for this comparison was not significant in our univariate analysis (Fig. 2).

Summary. Prospective memory improved up to 4 months later in this underpowered trial that was not placebo-controlled, on a non-validated measure, but only when baseline memory scores were taken into account. ${ }^{20}$

\section{Individual psychological interventions (one study)}

This lower-quality study (validity score: 3 ) found that an individual psychological intervention did not improve memory. Jean $e$ t $a l^{53}$ evaluated six individual 45 min sessions over 3 weeks, focusing on errorless learning of picture-name associations with spaced retrieval (see earlier). In the control condition, the pictures were presented without spaced retrieval. Participants were given written information about memory. The unvalidated primary outcomes evaluated the number of unknown faces (episodic memory) and famous names (semantic memory) matched correctly. There was no significant treatment effect on these measures in mixed-linear models $(F(2,35)=49.390$, $F(2,35)=11569)$, or on Mini-Mental State Examination (MMSE).

Summary. Six individual sessions of errorless learning and spaced retrieval did not improve prospective memory in one placebo-controlled, underpowered study where this was a primary outcome. $^{53}$

\section{Exercise}

Exercise has been associated with favourable effects on neuronal survivability and function, neuroinflammation, vascularisation, neuroendocrine response to stress and brain amyloid burden. It also improves cardiovascular health, which is associated with cognitive health. ${ }^{17}$

Group exercise programmes (two studies). Results from two studies comparing year-long, twice-weekly, group-based exercise programmes to active control conditions were mixed. In a very high-quality study (validity score: 5), van Uffelen et al ${ }^{38,54}$ compared a moderate-intensity walking programme to lowintensity relaxation, balance and flexibility exercises, and found no significant effect in any cognitive domain, including the primary outcome of immediate word recall (Fig. 2) or in quality of life. In a lower-quality study (validity score: 3; no primary outcomes specified), Suzuki et al ${ }^{42}$ compared groups involving circuit training and some outdoor walking with a control group who attended three health promotion classes. The intervention group improved in terms of MMSE score, immediate memory and verbal fluency (Table DS2).

Individual exercise programmes (three studies). None of these lower-quality studies specified primary outcomes. Busse et $a l^{26}$ (validity score: 3 ) evaluated 9 months of resistance exercises, for $1 \mathrm{~h}$ twice a week. Scores on a test of everyday memory (Rivermead Behaviour Memory Test) improved in the intervention group relative to the no-treatment controls, but CAMCog (Cambridge Cognition Examination) scores and digit span did not.

The remaining two studies had validity scores of 2 . Baker et $a l^{17}$ compared cognition in adults exercising less than $90 \mathrm{~min}$ weekly participating in a 6-month high-intensity aerobic exercise intervention or a stretching and balance exercise control. Each intervention was for $1 \mathrm{~h} 4$ days a week. The first eight sessions, and thereafter one session a week, were supervised. Adherence was monitored. Significant between-group effects, favouring the intervention, were reported on the Digit Symbol Substitution Test (DSST) (attention and processing speed), trail making test B and verbal fluency (Table DS2). For verbal fluency, effects were more apparent for category than letter fluency (letter, $P=0.20$, category, $P=0.03)$. Scherder et $a l^{41}$ compared: assisted walking for $30 \mathrm{~min}, 3$ days a week for 6 weeks; hand and face exercises for the same duration; and a control group, half of whom received additional social visits. The only significant between-group differences, all in favour of the interventions, were on the category fluency and trail making tests (Table DS2).

\section{Summary.}

(a) A very high-quality study found that memory, the primary outcome, did not improve with a year-long aerobic exercise group compared with a relaxation, balance and flexibility exercise active control group. ${ }^{38,54}$ A lower-quality study found that participants in a similar intervention improved on fluency, memory and global cognition relative to a health promotion control. ${ }^{42}$

(b) The studies of individual exercise studies were low quality and their results were inconsistent. ${ }^{17,26,41}$ Category fluency and trail-making test scores improved with individual aerobic exercise on secondary outcomes in two studies, of 6 weeks and 6 months duration, but no other cognitive outcomes improved in more than one study. 


\section{Pharmacological interventions}

\section{Cholinesterase inhibitors (nine studies)}

Three large studies compared donepezil $10 \mathrm{mg}$ daily to placebo, and results were inconsistent. The highest-quality one (validity score: 5) by Doody et al ${ }^{15}$ was a 48 -week study that included two primary outcome measures: ADAS-Cog, on which results favoured donepezil, and the Clinical Dementia Rating (CDR) on which there was no significant between-group difference (Fig. 2). On secondary outcomes, only patient global assessment differed significantly between groups, in favour of donepezil. The two other large studies were higher-quality studies (validity score: 4). Salloway et al $^{48}$ carried out a 24 -week, adequately powered study and there were no significant differences between donepezil and placebo on the primary outcomes, the New York University Paragraph Delayed Recall test or the Clinician Global Impression of Change (CGIC), or on any secondary outcomes except for the ADAS-Cog. Petersen et al ${ }^{45}$ found no significant difference between groups on conversion to Alzheimer's disease, the primary outcome (Fig. 3) or any other measures over 3 years. One small, lower-quality study (validity score: 1 ) that did not identify primary outcomes found that donepezil and antidepressant treatment improved immediate memory but not other cognitive outcomes, compared with antidepressants alone. ${ }^{27}$

Galantamine was investigated in three trials, and results on primary outcomes in the highest-quality trials were not significant. Winblad et $a l^{51}$ evaluated galantamine, titrated to $12 \mathrm{mg}$ twice daily in two large, high-quality, 24-month, placebocontrolled RCTs (validity scores 5 ). Neither reported a significant effect on the primary outcome, incident dementia (developing dementia in intervention $22.9 \% \quad v$. control group 22.6\%, $P=0.15,25.4 \%$ v. $31.2 \%, P=0.62)$. On secondary measures, statistical comparisons favoured galantamine in one of the two studies for global functioning (measured on the CDR) and attention (DSST). In a small, lower-quality trial, Koontz \& Baskys $^{22}$ only reported significant between-group differences on two subscales of the CANTAB, both measuring executive functioning (Table DS2).

One high-quality study (validity score: 5 ), by Feldman et al $^{31}$ compared 3-12 mg daily of rivastigmine and placebo. There were no significant differences between participants on any measures over 2 years, including the primary outcome, progression to Alzheimer's disease (Fig. 3).

Finally, Rozzini et $a l^{47}$ compared people receiving any cholinesterase inhibitor to those taking placebo after 1 year in a lower-quality study (validity score: 2 ). There were no significant between-group differences on any measures (Table DS4).

\section{Summary.}

(a) Incident Alzheimer's disease was not reduced in four, higherquality trials where this was the primary outcome - two evaluated galantamine, one donepezil, and one rivastigmine. ${ }^{31,45,51}$

(b) Donepezil improved global cognition in one high-quality trial where it was a primary outcome measure, ${ }^{15}$ and a second where it was a secondary outcome, ${ }^{48}$ but global cognition did not improve in the five other large, high-quality trials of cholinesterase inhibitors. ${ }^{31,45,47,51}$

(c) Donepezil did not improve global functioning in one trial where this was a primary outcome. ${ }^{15}$ Galantamine improved global functioning in one trial on a secondary outcome measure. $^{51}$

(d) Galantamine improved executive functioning and attention on secondary outcome measures in a single trial. ${ }^{51}$ (e) Donepezil as an adjunct to antidepressants improved immediate memory, also on a secondary outcome. ${ }^{27}$

\section{Piribedil (one study)}

Piribedil is a dopamine receptor agonist. Animal models have suggested acetylcholine release in hippocampi and the frontal cortex as a putative mechanism of action. Nagaraja \& Jayashree ${ }^{23}$ evaluated this over 3 months in a higher-quality trial (validity score: 4) with 30 in each group, all with a MMSE of 21-25; the primary outcome, response on the MMSE (predefined as a score of 26+), favoured piribedil (Fig. 5). Mean MMSE change from baseline also favoured the intervention group $(t=283, P<0.01)$. It was well tolerated.

Summary. Pirbidel improved cognition over 3 months on a primary outcome in one small placebo-controlled study. ${ }^{23}$

\section{Nicotine (one study)}

Brain nicotinic receptors are important for cognitive function. ${ }^{21}$ Newhouse et al ${ }^{21}$ compared transdermal nicotine (titrated to a $15 \mathrm{mg}$ patch/day) to placebo in a very high-quality study (validity score: 5 , with primary outcomes specified). Attention, measured on the Connors Cognitive Performance Test improved $(F=4.89$, $P=0.031$, effect size 078) but global functioning on the CGIC did not, on mixed-models repeated-measures analyses of variance. On secondary outcome measures, the treatment group showed less forgetting in between immediate and delayed recall than placebo $(F=4.42, \quad P=0.04)$, better delayed word recall $(F=5.92$, $P=0.018)$ and less anxiety on the older Adult Self Report worries and anxiety subscales $(F=3.48, P=0.04 ; F=3.14, P=0.05)$.

Summary. Nicotine patches improved attention, but not global functioning, over 6 months on primary outcomes in one, highquality study. ${ }^{21}$ Delayed recall and self-reported anxiety improved on secondary outcomes.

\section{Huannao Yicong (one study)}

Li et $a l^{28}$ evaluated this Chinese medicinal compound, which includes ginseng, in a study that identified primary outcomes but was low quality (validity score: 0 ). Increases or changes in hippocampal mitochondria have been proposed as mechanisms of action. Over 2 months, comparisons favoured the intervention on the primary outcome, response (improvement of $6+$ points) on the Cognitive Effect Index (CEI), which comprised the MMSE, Cognitive Capacity Screening Examination (CCSE) and a social functioning scale (Short-Form (SF)-36, Chinese version). We found that the mean difference in CEI scores between groups post-treatment was not significant (Table DS1). The analyses excluded people who did not take their medication.

Summary. Results in one low-quality trial were equivocal: more participants taking Huannao Yicong than placebo responded on a cognition and social functioning measure, but the mean difference between groups on this measure was not significant. ${ }^{28}$

\section{Gingko biloba (two studies)}

Results from these studies were inconsistent, but the highestquality trials suggested it is ineffective. Proposed mechanisms of action of Gingko biloba include increasing brain blood supply, reducing blood viscosity, modifying neurotransmitter systems, and reducing oxygen free radical density. ${ }^{56} \mathrm{~A}$ very high-quality study (validity score: 5 , published in two papers: deKosky et al ${ }^{35}$ 
and Snitz et $\mathrm{al}^{57}$ ) found that $240 \mathrm{mg}$ daily, taken for a median of 6.1 years, did not reduce incident dementia or Alzheimer's disease. In a lower-quality (validity score: 3), 6-month study that was not placebo controlled, Zhao et $a l^{25}$ reported that participants prescribed $56.7 \mathrm{mg}$ daily Gingko biloba performed better than those receiving no treatment on nonsense picture recognition and logical memory tests (Fig. 3).

Summary. On primary outcomes, $240 \mathrm{mg}$ daily Gingko biloba did not reduce incident dementia in a very high-quality trial over 6 years; ${ }^{35,57}$ whereas $56.7 \mathrm{mg}$ daily improved cognition in a second trial compared with usual treatment. ${ }^{25}$

\section{NSAIDs (two studies)}

Non-steroidal anti-inflammatory drugs reduce brain neurotoxic inflammatory responses, so could improve cognition. ${ }^{58}$ Thal et $a l,{ }^{49}$ in a very high-quality large study (validity score: 5 ), found significantly more incident cases of Alzheimer's disease over 4 years in participants randomised to $25 \mathrm{mg}$ daily of rofecoxib (a COX-2 inhibitor) than those taking placebo (Fig. 4). There was no significant difference between groups on secondary outcomes. In 2003, rofecoxib was withdrawn due to cerebrovascular and cardiovascular side-effects.

Gomez-Isla et al ${ }^{44}$ evaluated $900 \mathrm{mg}$ a day of triflusal, a COX-1 and COX-2 inhibitor in a lower-quality study (validity score: 3 ), which was terminated early. It found no significant difference between groups on the primary cognitive outcome, ADAS-Cog (Fig. 2). The only significant finding on secondary outcomes was a lower rate of conversion to Alzheimer's disease in the intervention group ( $\mathrm{HR}=210,95 \% \mathrm{CI} 1.10-4.01, P=0.024)$.

\section{Summary.}

(a) Rofecoxib increased incident cases of Alzheimer's disease in one very high-quality study on a primary outcome. ${ }^{49}$

(b) One trial of triflusal reported no significant effect on cognition on a primary outcome measure, although it was associated with a reduced risk of conversion to Alzheimer's disease on a secondary outcome. ${ }^{44}$

\section{B vitamins (two studies)}

Higher homocysteine plasma concentrations are associated with cognitive impairment; levels are decreased by B vitamins. Two placebo-controlled trials investigated the effectiveness of $\mathrm{B}$ vitamins (folic acid, $\mathrm{B}_{12}$ and $\mathrm{B}_{6}$ ). In a very high-quality study (validity score: 5 and primary outcome specified), van Uffelen et $a l^{38}$ found no significant difference on the primary outcome of immediate memory over 6 months. On secondary outcomes, the group taking vitamins performed better than the placebo group on the DSST (attention and processing speed; longitudinal regression, coefficient not given, $P=0.02$ ). De Jager et $a l^{30}$ found in a lower-quality (validity score: 3 ), 2-year study that executive functioning improved relative to placebo (Table DS2).

Summary. Immediate memory did not improve in a high-quality study in which this was primary outcome. ${ }^{38}$ Out of numerous secondary measures, attention improved in one trial and executive functioning in another, ${ }^{30}$ so results were inconsistent.

\section{Vitamin E (two studies)}

One large, higher-quality trial (validity score: 4) reported by Petersen et al ${ }^{45}$ found no significant treatment effect of vitamin E (2000 IU) on the primary outcome measure, progression to
Alzheimer's disease or on a range of secondary outcomes over 3 years. Zhou et al ${ }^{50}$ reported in a lower-quality study (validity score: 1) that participants receiving vitamin E $500 \mathrm{mg}$ daily improved $v$. placebo on picture recognition (Table DS2).

\section{Summary.}

(a) Vitamin E did not reduce incident dementia in one highquality study on a primary outcome. ${ }^{45}$

(b) In a lower-quality study $500 \mathrm{mg}$ daily was associated with improvement in picture recognition, a secondary outcome..$^{50}$

\section{Omega-3 polyunsaturated fatty acids (two studies)}

Docosahexaenoic acid (DHA) and eicosapentaenoic acid (EPA) are dietary polyunsaturated fatty acids (PUFA), which have structural and functional roles in the brain. Both these studies had validity scores of 3 . Chiu et $a l^{29}$ found that, as primary outcomes, ADAS-Cog improved over 6 months in people taking $1080 \mathrm{mg}$ EPA and $720 \mathrm{mg}$ DHA $v$. placebo after adjusting for age, gender and education, but no differences were reported on the Clinician's Interview-Based Impression of Change (CIBICplus, global functioning). When we calculated SMD for ADAS$\mathrm{Cog}$ at follow-up between groups, there were no significant differences (Table DS1).

Sinn $e$ t $a l^{34}$ in a small study compared groups receiving EPA-rich fish oil (1670 mg EPA and $160 \mathrm{mg}$ DHA) and DHA-rich fish oil (1550 mg DHA and $400 \mathrm{mg}$ EPA) with a placebo group. Using a linear-mixed model analysis, letter fluency scores significantly improved over 6 months in the DHA group $v$. placebo, and depressive symptoms, measured using the Geriatric Depression Score, were reduced in both groups (Table DS2).

\section{Summary.}

(a) Cognition improved on a primary outcome in one study, but only after adjusting for age, gender and education. ${ }^{29}$

(b) Verbal fluency improved with DHA-rich fish oil and depressive symptoms were reduced by DHA- and EPA-rich oil after 6 months in a single small study on secondary outcome measures. ${ }^{34}$

\section{Interventions evaluated in single trials without primary outcomes}

Ten different interventions have been evaluated in single trials, not specifying one or two primary outcomes (Table DS2). Three were higher-quality trials (validity scores $4+$ ). The first found that transcutaneous electrical nerve stimulation (TENS) treatment reduced activities of daily living impairment and depression over 6 weeks in the only trial we reviewed that did not measure cognition. ${ }^{39}$ The second trial found that in 3-month trials, memantine improved information processing speed but not cognition. ${ }^{52}$ The third found that a nutritional supplement composed of: DHA $720 \mathrm{mg}$, EPA $286 \mathrm{mg}$, vitamin E $16 \mathrm{mg}$, soy phospholipids $160 \mathrm{mg}$, tryptophan $95 \mathrm{mg}$ and melatonin $5 \mathrm{mg}^{40}$ improved cognition.

Fluoxetine, ${ }^{43}$ Shenyin oral liquid, ${ }^{50}$ Ginseng, ${ }^{37}$ Wuzi Yanzong, ${ }^{36}$ grape juice, ${ }^{32}$ Green tea $^{33}$ and lithium ${ }^{59}$ were ineffective in single, lower-quality trials.

\section{Discussion}

\section{Main findings}

Our most striking finding is the lack of good-quality evidence except in the pharmacological trials. These enable us to more 
confidently reject cholinesterase inhibitors as useful in preventing conversion of MCI to dementia, and to confirm NICE guidance that cholinesterase inhibitors should not be prescribed clinically for MCI. ${ }^{4}$ The only non-pharmacological intervention for which we found preliminary evidence, in a single, placebo-controlled trial on co-primary outcomes, was a heterogeneous group programme of memory training, reminiscence and cognitive stimulation, recreation and social interaction, which improved cognition over 6 months. There was equivocal evidence that a group intervention for families might improve prospective memory from a trial that was not placebo controlled. We also found replicated evidence on secondary outcomes that category fluency improved with individual aerobic exercise programmes, of 6 weeks and 6 months duration, and delayed recall improved in two studies evaluating computerised cognitive training programmes. These latter studies had multiple secondary outcomes, thus increasing the possibility of a chance finding and the clinical benefit of isolated improvements in these domains is unclear. Most studies were underpowered and lack of evidence of efficacy is not evidence of lack of efficacy.

In pharmacological studies, donepezil improved cognition over a year in two trials, one on a primary outcome, but in general the evidence from seven studies of cholinesterase inhibitors was not promising. The strongest evidence we found was that cholinesterase inhibitors ${ }^{31,51,45}$ did not reduce the incidence of dementia. Given the safety concerns around the use of cholinesterase inhibitors in $\mathrm{MCI}^{8}$ we think that trials of alternative therapeutic agents are now needed.

Piribedil, a dopamine agonist was effective on a cognitive primary outcome in one study. ${ }^{23}$ However, the criteria for MCI were not strict and the authors acknowledge that some of the participants may have had dementia. Nicotine patches improved attention on a primary outcome over 6 months, and also verbal recall on a secondary outcome; and we found equivocal evidence that Huannao Yicong, a Chinese herbal preparation, may improve cognition and social functioning.

It is disappointing that we did not find stronger evidence of efficacy, but nonetheless some of the interventions included warrant further investigation. It is unclear why there have been no further trials of piribedil in MCI since the positive trial reported in 2001; a trial of piribedil in people with Parkinson's disease has not yet reported (ClinicalTrials.gov identifier: NCT01007864). The effectiveness of Huannao Yicong in one trial, albeit of low validity, could indicate that further exploration of Chinese medicine treatments of MCI may be fruitful. There was limited evidence that exercise therapies improved executive functioning. Resistance training, walking and aerobic exercises may well differ in their effects, and given the positive impact of exercise on general health this would also be an interesting area for future study.

\section{Limitations}

This is one of the first comprehensive reviews of all treatments evaluated for MCI. Methodological challenges for MCI trials include deciding inclusion criteria. Nearly two-thirds of studies used Petersen criteria. Some of the studies only included participants with amnestic MCI, whereas others included other subtypes so even within those using Petersen criteria, the target groups were heterogeneous. Some people with MCI have prodromal Alzheimer's disease or will progress to vascular or other subtypes of dementia. Only two-thirds of people with MCI progress to dementia in their lifetime ${ }^{60}$ limiting the power of secondary prevention studies that recruit MCI populations. The heterogeneity and instability of the MCI diagnosis militate against finding positive results in MCI trials. It is interesting that although in the trial reported by Petersen et al, ${ }^{45}$ vitamin E did not prevent Alzheimer's disease in people with amnestic MCI overall, it was effective at doing so among carriers of one or more apolipoprotein Eå4 alleles, perhaps because this is a more homogeneous group, more likely to have prodromal Alzheimer's disease. Availability of biomarkers may enable future trials to recruit participants according to disease process rather than clinical deficits. For example, trials of pharmacological agents targeting people with early Alzheimer's disease may recruit people with amnestic MCI and probable Alzheimer's disease. ${ }^{61}$ Biomarkers may also allow participants to be recruited earlier in the disease process, at the stage of subjective memory impairment, which usually precedes MCI. By the time MCI develops the pathological process may be too advanced for treatments to be preventive, perhaps because the brain is by this point very vulnerable to other comorbidities that lead to a dementia, even though progression of the original pathology is halted.

A second challenge is deciding on a primary outcome. 'Conversion' trials are difficult to power adequately as only $10 \%$ of people with MCI convert every year to dementia, and this rate seems to be lower in RCTs. ${ }^{31}$ Incident dementia is often the primary outcome as dementia prevention is a clear goal, but Schneider has suggested it is a problematic end-point because many participants would be on the cusp of dementia and dementia onset is influenced by numerous biological and environmental factors. ${ }^{61}$ We prioritised placebo-controlled trials, because this evidence is most directly applicable to current practice. There are no evidence-based interventions for MCI and most people with it receive no active treatment. We included a broad range of clinical outcomes, but excluded studies evaluating subjective experiences of memory or biological markers. We included papers in all languages, but only searched English language databases. We planned to meta-analyse findings from three or more studies, but in practice only donepezil was evaluated in more than two studies, and this could not be meta-analysed as the required data were unavailable from one study.

\section{Implications}

There is no evidence, replicated on primary outcomes, that any intervention is effective for MCI for the outcomes studied. Results for cholinesterase inhibitors in MCI, the most widely studied intervention, are unpromising. More high-quality RCTs are urgently needed. This review would support further trials of a heterogeneous group psychological intervention and a dopamine agonist as interventions targeting cognition.

\section{Claudia Cooper, MRCPsych, PhD, Ryan Li, BSC, Mental Health Sciences Unit, University College London, UK; Constantine Lyketsos, MD, MHS, Department of Psychiatry and Behavioral Sciences, Johns Hopkins School of Medicine and Johns Hopkins Bayview, Johns Hopkins Medicine, Baltimore, Maryland, USA; Gill Livingston, FRCPsych, MD, Mental Health Sciences Unit, University College London, UK}

Correspondence: Claudia Cooper, Mental Health Sciences Unit, University College London, Holborn Union Building, Highgate Hill, London N19 5LW, UK. Email: c.cooper@ucl.ac.uk

First received 12 Feb 2013, final revision 16 Apr 2013, accepted 22 Apr 2013

\section{References}

1 Petersen RC. Mild cognitive impairment as a diagnostic entity. J Intern Med 2004; 256: 183-94.

2 Lopez OL, Kuller LH, Becker JT, Dulberg C, sweet RA, Gach HM et al. Incidence of dementia in mild cognitive impairment in the cardiovascular health study cognition study. Arch Neurol 2007; 64: 416-20. 
3 Tschanz JT, Welsh-Bohmer KA, Lyketsos CG, Corcoran C, Green RC, Hayden K, et al. Conversion to dementia from mild cognitive disorder - the Cache County Study. Neurology 2006; 67: 229-34.

4 National Institute for Health and Clinical Excellence. Dementia: Supporting People with Dementia and their Carers in Health and Social Care. Clinical Guideline 42. NICE, 2006

5 Jorm $A F$, Korten $A E$, Henderson AS. The prevalence of dementia - a quantitative integration of the literature. Acta Psychiatr Scand 1987; 76: 465-79.

6 Raschetti R, Albanese E, Vanacore N, Maggini M. Cholinesterase inhibitors in mild cognitive impairment: a systematic review of randomised trials. PLOS Med 2007; 4: e338.

7 Birks J, Flicker L. Donepezil for mild cognitive impairment. Cochrane Database Syst Rev 2006; 3: CD006104.

8 Loy C, Schneider L. Galantamine for Alzheimer's disease and mild cognitive impairment. Cochrane Database Syst Rev 2006; 1: CD001747.

9 Martin M, Clare L, Altgassen AM, Cameron MH, Zehnder F. Cognition-based interventions for healthy older people and people with mild cognitive impairment. Cochrane Database Syst Rev 2011; 1: CD006220.

10 Gates NJ, Sachdev PS, Fiatarone Singh MA, Valenzuela M. Cognitive and memory training in adults at risk of dementia: a systematic review. BMC Geriatr 2011; 11: 55 .

11 Jean L, Bergeron ME, Thivierge S, Simard M. Cognitive intervention programs for individuals with mild cognitive impairment: systematic review of the literature. Am J Geriatr Psychiatry 2010; 18: 281-96.

12 Simon SS, Yokomizo JE, Bottino CM. Cognitive intervention in amnestic mild cognitive impairment: a systematic review. Neurosci Biobehav Rev 2012; 36 1163-78.

13 Stott J, Spector A. A review of the effectiveness of memory interventions in mild cognitive impairment (MCl). Int Psychogeriatr 2011; 23: 526-38.

14 Unverzagt FW, Kasten L, Johnson KE, Rebok GW, Marsiske M, Koepke KM, et al. Effect of memory impairment on training outcomes in ACTIVE. J Internat Neuropsychol Soc 2007; 13: 953-60.

15 Doody RS, Ferris SH, Salloway S, Sun Y, Goldman R, Watkins WE, et al. Donepezil treatment of patients with $\mathrm{MCl}$ : a 48-week randomized placebo-controlled trial. Neurology 2009; 72: 1555-61.

16 StatsDirect. Stats Direct Statistical Software. StatsDirect, 2012.

17 Baker LD, Frank LL, Foster-Schubert K, Green PS, Wilkinson CW, McTiernan A et al. Effects of aerobic exercise on mild cognitive impairment: a controlled trial. Arch Neurol 2010; 67: 71-9.

18 Barnes DE, Yaffe K, Belfor N, Jagust WJ, DeCarli C, Reed BR, et al. Computer based cognitive training for mild cognitive impairment: results from a pilot randomized, controlled trial. Alzheimer Dis Assoc Disord 2009; 23: 205-10.

19 Buschert VC, Friese U, Teipel SJ, Schneider P, Merensky W, Rujescu D, et al. Effects of a newly developed cognitive intervention in amnestic mild cognitive impairment and mild Alzheimer's disease: a pilot study. J Alzheimers Dis 2011; 25: 679-94.

20 Kinsella GJ, Mullaly E, Rand E, Ong B, Burton C, Price S, et al. Early intervention for mild cognitive impairment: a randomised controlled trial. J Neurol Neurosurg Psychiatry 2009; 80: 730-6.

21 Newhouse $P$, Kellar $K$, Aisen $P$, White $H$, Wesnes $K$, Coderre $E$, et al. Nicotine treatment of mild cognitive impairment: a 6-month double-blind pilot clinical trial. Neurology 2012; 78: 91-101.

22 Koontz J, Baskys A. Effects of galantamine on working memory and global functioning in patients with mild cognitive impairment: a double-blind placebo-controlled study. Am J Alzheimers Dis Other Demen 2005; 20 295-302.

23 Nagaraja D, Jayashree S. Randomized study of the dopamine receptor agonist piribedil in the treatment of mild cognitive impairment. Am J Psychiatry 2001; 158: 1517-9.

24 Troyer AK, Murphy KJ, Anderson ND, Moscovitch M, Craik Fl. Changing everyday memory behaviour in amnestic mild cognitive impairment: a randomised controlled trial. Neuropsychol Rehabil 2008; 18: 65-88.

25 Zhao MX, Dong ZH, Yu ZH, Xiao SY, Li YM. Effects of Ginkgo biloba extract in improving episodic memory of patients with mild cognitive impairment: a randomized controlled trial [in Chinese]. Zhong Xi Yi Jie He Xue Bao 2012 10: $628-34$

26 Busse AL, Filho JW, Magaldi RM, Coelho VA, Melo AC, Betoni RA, et al. Effects of resistance training exercise on cognitive performance in elderly individuals with memory impairment: results of a controlled trial. Einstein 2008; 6: 402-7.

27 Pelton GH, Harper OL, Tabert MH, Sackeim HA, Scarmeas N, Roose SP, et al. Randomized double-blind placebo-controlled donepezil augmentation in antidepressant-treated elderly patients with depression and cognitive impairment: a pilot study. Int J Geriatr Psychiatry 2008; 23: 670-6.
28 Li H, Yao MJ, Zhao WM, Guan J, Cai LL, Cui L. A randomized, controlled, double-blind trial of Huannao Yicong capsule in senile patients with mild cognitive impairment [in Chinese]. Zhong Xi Yi Jie He Xue Bao 2008; 6: 25-31.

29 Chiu CC, Su KP, Cheng TC, Liu HC, Chang CJ, Dewey ME, et al. The effects of omega-3 fatty acids monotherapy in Alzheimer's disease and mild cognitive impairment: a preliminary randomized double-blind placebo-controlled study. Prog Neuropsychopharmacol Biol Psychiatry 2008; 32: 1538-44.

30 de Jager CA, Oulhaj A, Jacoby R, Refsum H, Smith AD. Cognitive and clinical outcomes of homocysteine-lowering B-vitamin treatment in mild cognitive impairment: a randomized controlled trial. Int J Geriatr Psychiatry 2012; 27 592-600.

31 Feldman $\mathrm{HH}$, Ferris S, Winblad B, Sfikas $\mathrm{N}$, Mancione $\mathrm{L}$, He $\mathrm{Y}$, et al. Effect of rivastigmine on delay to diagnosis of Alzheimer's disease from mild cognitive impairment: the InDDEx study. Lancet Neurol 2007; 6: 501-12.

32 Krikorian R, Nash TA, Shidler MD, Shukitt-Hale B, Joseph JA. Concord grape juice supplementation improves memory function in older adults with mild cognitive impairment. Br J Nutr 2010; 103: 730-4.

33 Park SK, Jung IC, Lee WK, Lee YS, Park HK, Go HJ, et al. A combination of green tea extract and I-theanine improves memory and attention in subjects with mild cognitive impairment: a double-blind placebo-controlled study. J Med Food 2011; 14: 334-43.

34 Sinn N, Milte CM, Street SJ, Buckley JD, Coates AM, Petkov J, et al. Effects of n-3 fatty acids, EPA v. DHA, on depressive symptoms, quality of life, memory and executive function in older adults with mild cognitive impairment: a 6-month randomised controlled trial. Br J Nutr 2012; 107: 1682-93.

35 DeKosky ST, Williamson JD, Fitzpatrick AL, Kronmal RA, Ives DG, Saxton JA, et al. Ginkgo biloba for prevention of dementia: a randomized controlled trial. JAMA 2008; 300: 2253-62.

36 Fu H, Wang XM, Liu GX. Effects of modified Wuzi Yanzong Granule on memory ability and volume of hippocampus measured by MRI in patients with mild cognitive impairment [in Chinese]. Zhongguo Zhong Xi Yi Jie He Za Zhi 2006; 26: 1066-9.

37 Tian JZ, Zhu AH, Zhong J. A follow-up study on a randomized, single-blind control of King's Brain pills in treatment of memory disorder in elderly people with $\mathrm{MCl}$ in a Beijing community [in Chinese]. Zhongguo Zhong Yao Za Zhi 2003; 28: 987-91.

38 van Uffelen JG, Chinapaw MJ, van MW, Hopman-Rock M. Walking or vitamin $\mathrm{B}$ for cognition in older adults with mild cognitive impairment? A randomised controlled trial. Br J Sports Med 2008; 42: 344-51.

39 Luijpen MW, Swaab DF, Sergeant JA, Scherder EJ. Effects of transcutaneous electrical nerve stimulation (TENS) on self-efficacy and mood in elderly with mild cognitive impairment. Neurorehabil Neural Repair 2004; 18: 166-75.

40 Rondanelli M, Opizzi A, Faliva M, Mozzoni M, Antoniello N, Cazzola R, et al. Effects of a diet integration with an oily emulsion of DHA-phospholipids containing melatonin and tryptophan in elderly patients suffering from mild cognitive impairment. Nutr Neurosci 2012; 15: 46-54

41 Scherder EJ, Van PJ, Deijen JB, Van Der Knokke S, Orlebeke JF, Burgers I, et al. Physical activity and executive functions in the elderly with mild cognitive impairment. Aging Ment Health 2005; 9: 272-80.

42 Suzuki T, Shimada H, Makizako H, Doi T, Yoshida D, Tsutsumimoto K, et al. Effects of multicomponent exercise on cognitive function in older adults with amnestic mild cognitive impairment: a randomized controlled trial. $B M C$ Neurology 2012; 12: 128

43 Mowla A, Mosavinasab M, Pani A. Does fluoxetine have any effect on the cognition of patients with mild cognitive impairment? A double-blind placebo-controlled, clinical trial. J Clin Psychopharmacol 2007; 27: 67-70.

44 Gomez-Isla T, Blesa R, Boada M, Clarimon J, Del ST, Domenech G, et al. A randomized, double-blind, placebo controlled-trial of triflusal in mild cognitive impairment: the TRIMCI study. Alzheimer Dis Assoc Disord 2008; 22: 21-9.

45 Petersen RC, Thomas RG, Grundman M, Bennett D, Doody R, Ferris S, et al. Vitamin $E$ and donepezil for the treatment of mild cognitive impairment. N Engl J Med 2005; 352: 2379-88.

46 Rapp S, Brenes G, Marsh AP. Memory enhancement training for older adults with mild cognitive impairment: a preliminary study. Aging Ment Health 2002; 6: 5-11.

47 Rozzini L, Costardi D, Chilovi BV, Franzoni S, Trabucchi M, Padovani A Efficacy of cognitive rehabilitation in patients with mild cognitive impairment treated with cholinesterase inhibitors. Int J Geriatr Psychiatry 2007; 22: 356-60.

48 Salloway S, Ferris S, Kluger A, Goldman R, Griesing T, Kumar D, et al. Efficacy of donepezil in mild cognitive impairment: a randomized placebo-controlled trial. Neurology 2004; 63: 651-7.

49 Thal LJ, Ferris SH, Kirby L, Block GA, Lines CR, Yuen E, et al. A randomized, double-blind, study of rofecoxib in patients with mild cognitive impairment. Neuropsychopharmacology 2005; 30: 1204-15 
50 Zhou RQ, Lin SM, Yuan Q. Clinical study on effect of Shenyin Oral Liquid in treating mild cognitive impairment [in Chinese]. Zhongguo Zhong Xi Yi Jie He Za Zhi 2007; 27: 793-5.

51 Winblad B, Gauthier S, Scinto L, Feldman H, Wilcock GK, Truyen L et al. Safety and efficacy of galantamine in subjects with mild cognitive impairment. Neurology 2008; 70: 2024-35.

52 Ferris S, Schneider L, Farmer M, Kay G, Crook T. A double-blind, placebocontrolled trial of memantine in age-associated memory impairment (memantine in AAMI). Int J Geriatr Psychiatry 2007; 22: 448-55.

53 Jean L, Simard M, Wiederkehr S, Bergeron ME, Turgeon Y, Hudon C, et al. Efficacy of a cognitive training programme for mild cognitive impairment: results of a randomised controlled study. Neuropsychol Rehabil 2010; 20 377-405.

54 van Uffelen JG, Chin APMJ, Hopman-Rock M, van Mechelen W. The effect of walking and vitamin B supplementation on quality of life in communitydwelling adults with mild cognitive impairment: a randomized, controlled trial. Qual Life Res 2007; 16: 1137-46.

55 Finn $\mathrm{M}$, McDonald S. Computerised cognitive training for older persons with mild cognitive impairment: a pilot study using a randomised controlled trial design. Brain Impair 2011; 12: 187-99.
56 Birks J, Evans JG. Ginkgo biloba for cognitive impairment and dementia. Cochrane Database Syst Rev 2009; 1: CD003120.

57 Snitz BE, O'Meara ES, Carlson MC, Arnold AM, Ives DG, Rapp SR, et al Ginkgo biloba for preventing cognitive decline in older adults: a randomized trial. JAMA 2009; 302: 2663-70.

58 Weggen S, Eriksen JL, Das $\mathrm{P}$, Sagi SA, Wang R, Pietrzik CU, et al. A subse of NSAIDs lower amyloidogenic Abeta42 independently of cyclooxygenase activity. Nature 2001; 414: 212-6.

59 Forlenza OV, Diniz BS, Radanovic M, Santos FS, Talib LL, Gattaz WF. Disease-modifying properties of long-term lithium treatment for amnestic mild cognitive impairment: randomised controlled trial. Br J Psychiatry 2011; 198: $351-6$.

60 Busse A, Angermeyer MC, Riedel-Heller SG. Progression of mild cognitive impairment to dementia: a challenge to current thinking. $\mathrm{Br} J$ Psychiatry 2006; 189: 399-404.

61 Schneider LS. The potential and limits for clinical trials for early Alzheimer's disease and some recommendations. J Nutr Health Aging 2010; 14: 295-8.

man

\section{fallen \\ Daswanth}

Daswanth was born in India in 1560. He was the son of a palanquin bearer who transported people belonging to social classes many levels higher than his own. Despite this humble background, Daswanth's gifts were discovered by the Mughal emperor Akbar, a connoisseur of art, who would often canvass his domain looking for potential talent to beautify his courthouses and other public buildings. Emperor Akbar had noticed Daswanth's talent and sent him to the local painting master for a period of intensive training.

Rumours circulated about a disquieting side to the young painter. His behaviour was thought to be impulsive, even irrational at times. Images arise of a wildly eccentric artist depicting his foreboding visions with ominous intensity. Among the most vivid of Daswanth's surviving works is 'A Night Assault on the Pandava Camp'. This morbid masterpiece depicts a mythical spirit feasting on the blood of battle victims. In the bloody scene, a sultry female demon delights in her necklace of decapitated heads and garments consisting of the skins of slaughtered animals. Adding to the chaos is a certain stylistic distortion, in which lines overlap and lead to spatial ambivalence.

The irrational and alarming qualities of Daswanth's work began to surface more often in his behaviour. Whatever could be done for him in this era, one can only conjecture. What is certain is that the era in which he lived far pre-dated the advent of modern psychiatry, and there was therefore nothing to prevent his fall into a maelstrom of self-destruction.

Daswanth's demise would only serve to reinforce his 'madman' image, as at the mere age of 24 he fatally stabbed himself At present, few paintings can be confidently attributed to Daswanth. Of these, most are found in a richly illustrated Indian manuscript produced during the late 16 th century. 